\title{
UCRL-PROC-219279
}

LAW RENCE LIVERMORE N A T IO N A L LABORATORY

\section{EUV testing of multilayer mirrors: critical issues}

S. B. Hill, I. Ermanoski, S. Grantham, C. Tarrio, T. B. Lucatorto, T. E. Madey, S. Bajt, M. Chandhok, P. Yan, O. Wood, S. Wurn, N. V. Edwards

February 24, 2006

SPIE Microlithography

San Jose, CA, United States

February 19, 2006 through February 24, 2006 
This document was prepared as an account of work sponsored by an agency of the United States Government. Neither the United States Government nor the University of California nor any of their employees, makes any warranty, express or implied, or assumes any legal liability or responsibility for the accuracy, completeness, or usefulness of any information, apparatus, product, or process disclosed, or represents that its use would not infringe privately owned rights. Reference herein to any specific commercial product, process, or service by trade name, trademark, manufacturer, or otherwise, does not necessarily constitute or imply its endorsement, recommendation, or favoring by the United States Government or the University of California. The views and opinions of authors expressed herein do not necessarily state or reflect those of the United States Government or the University of California, and shall not be used for advertising or product endorsement purposes. 


\title{
EUV testing of multilayer mirrors: critical issues
}

\author{
S. B. Hill ${ }^{\mathrm{a}}$, I. Ermanoski ${ }^{\mathrm{a}}$, S. Grantham ${ }^{\mathrm{a}}$, C. Tarrio ${ }^{\mathrm{a}}$, T. B. Lucatorto ${ }^{\mathrm{a}}$, T. E. Madey ${ }^{\mathrm{b}}$, S. Bajt $^{\dagger c}$,

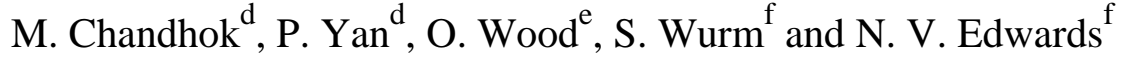 \\ ${ }^{\mathrm{a}}$ NIST, 100 Bureau Drive, Stop 8411, Gaithersburg, MD 20853-8411 \\ ${ }^{\mathrm{b}}$ Rutgers University, 136 Frelinghuysen Rd.,Piscataway, NJ 08854-8019 \\ c Lawrence Livermore National Laboratory, 7000 East Avenue, L-210, Livermore, CA 94550 \\ dintel Corporation, 2200 Mission College Blvd., Santa Clara, CA 95054 \\ e SEMATECH, 255 Fuller Road, suite 309, Albany, NY 12203 \\ ${ }^{\mathrm{f}}$ SEMATECH, 2706 Montopolis Dr., Austin, TX 78741-6499
}

\begin{abstract}
.
Recently, while performing extensive EUV irradiation endurance testing on Ru-capped multilayer mirrors in the presence of elevated partial pressures of water and hydrocarbons, NIST has observed that the amount of EUV-induced damage actually decreases with increasing levels of water vapor above $\sim 5 \times 10^{-7}$ Torr. It is thought that the admitted water vapor may interact with otherwise stable, condensed carbonaceous species in an UHV vacuum system to increase the background levels of simple gaseous carbon-containing molecules. Some support for this hypothesis was demonstrated by observing the mitigating effect of very small levels of simple hydrocarbons with the intentional introduction of methyl alcohol in addition to the water vapor. It was found that the damage rate decreased by at least an order of magnitude when the partial pressure of methyl alcohol was just one percent of the water partial pressure. These observations indicate that the hydrocarbon components of the vacuum environment under actual testing conditions must be characterized and controlled to $10^{-11}$ Torr or better in order to quantify the damage caused by high levels of water vapor. The possible effects of exposure beam size and out-of-band radiation on mirror lifetime testing will also be discussed.
\end{abstract}

Keywords: extreme ultraviolet; lithography; reflectometry; EUV optics; ruthenium films; lifetime testing

\section{INTRODUCTION}

Commercialization of extreme-ultraviolet lithography (EUVL) presents a number of significant technical challenges, including pushing metrology to new limits. The Photon Physics Group of the National Institute of Standards and Technology (NIST) has been involved in developing and providing EUVL metrology for almost two decades. In fact, the most mature program, EUV detector calibration, ${ }^{1}$ has been operating at the Synchrotron Ultraviolet Radiation Facility (SURF III) storage ring since well before the birth of the EUVL effort. In 1988 we began a program for the measurement of reflectivities of EUV multilayer (ML) optics. ${ }^{2}$ Most recently we have constructed a beamline dedicated to long-term studies of ML lifetimes in a controlled environment. ${ }^{3}$

The requisite ML lifetime is 30,000 hours, or about 3.5 years of continuous operation without a decrease in reflectivity of more than a few percent. With a predicted EUVL insertion point of about 2010, developers cannot test MLs for that period of time, necessitating the development of reliable accelerated testing methods. In this paper, we will describe our program and offer results from selected lifetime testing experiments.

\footnotetext{
${ }^{\dagger}$ This work was performed under the auspices of the U.S. Department of Energy by University of California Lawrence Livermore National Laboratory under contract No. W-7405-Eng-48
} 


\section{MULTILAYER ENDURANCE TESTING}

\subsection{The optics lifetime problem for EUV lithography}

EUV lithography employs a set of reflective optics to project the mask image onto the resist-covered wafer. The EUV mirrors are coated with an extremely precise ML coating consisting of 40 or more MoSi bilayers deposited with a $\sim 7$-nm period. Reflectivity approaching $70 \%$ is achieved through the constructive interference of the light rays with wavelength near $13.5 \mathrm{~nm}$ that are reflected from each of the appropriately spaced bilayers

The EUV mirrors are housed in a vacuum chamber called the projection optics (PO) box. Because baking the PO box would damage the mirrors by accelerating inter-diffusion of the MoSi bilayers and would destroy the precise alignment of the mirrors, the ambient background will contain residual water and hydrocarbon vapors. These trace gases can adsorb onto the optic surface and be fragmented by the energetic (91.8 eV) EUV photons or secondary electrons, producing reactive species. Oxygen atoms from dissociated water molecules can oxidize the mirror surface, while carbon from cracked hydrocarbon molecules can form a graphitic overlayer. An intensive effort is underway to develop a cap layer to protect the mirror surface from oxidation and carbon buildup because both cause undesirable reflectivity loss. The work presented here was performed using the first promising capping layer of Ru developed and fabricated at Lawrence Livermore National Labs. ${ }^{4}$ NIST's contribution to the effort is the provision of long-term EUV exposure testing under vacuum conditions with controlled amounts of water vapor and carbonaceous contaminants.

\subsection{EUV exposure facilities at NIST}

A schematic of one of the two endurance testing beamlines on SURF III is shown in figure 1. The output of the synchrotron is collected by a near-normal-incident ML mirror placed approximately $4 \mathrm{~m}$ from the storage ring tangent point. This ML is designed to reflect EUV radiation with a 10-degree angle of incidence in the bandwidth $13.1 \mathrm{~nm}$ to $13.6 \mathrm{~nm}$ with approximately $50 \%$ efficiency. The resulting focal spot is $\sim 1.5 \mathrm{~m}$ downstream and has a Gaussian intensity distribution with a time-averaged peak of $\sim 5 \mathrm{~mW} / \mathrm{mm}^{2}$ and a full-width half-max of $\sim 650 \mu \mathrm{m}$. The focusing optic also efficiently reflects the visible and IR light from the synchrotron. A $200 \mathrm{~nm}$-thick beryllium filter completely blocks this long-wavelength light while transmitting 50-60\% of the EUV radiation. More importantly, this filter also serves as a physical barrier separating the exposure chamber from the rest of the beamline. It is essential that the synchrotron and the focusing mirror not be exposed to the high levels of water and hydrocarbon vapors present in the

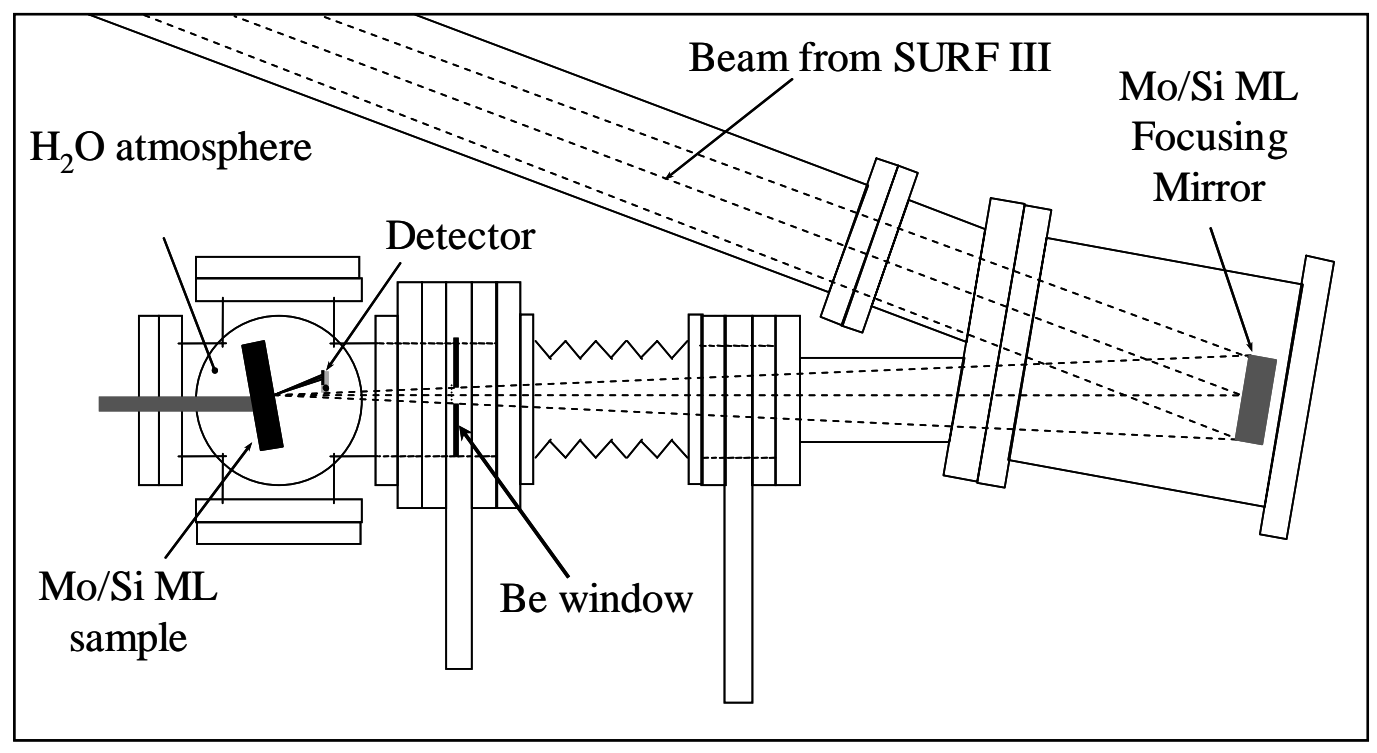

Figure 1. Schematic of the end-station on the SURF III synchrotron for endurance testing of EUV optics at NIST 
exposure chamber during most endurance tests. Since thermal diffusion of the ML structure would ruin the mirror to be tested, the exposure chamber can not be baked once the sample is installed. Instead the exposure chamber was constructed to meet UHV specifications and was pre-baked up to $150^{\circ} \mathrm{C}$. To minimize contamination, the chamber is slightly over-pressured with dry $\mathrm{N}_{2}$ to maintain a flow while new samples are loaded. In order to accelerate sample aging during testing, the partial pressure of water vapor in the exposure chamber can be varied between the base pressure of $<1 \times 10^{-9}$ Torr and $5 \times 10^{-6}$ Torr, which is considerably greater than the expected base pressure in a stepper. Similar pressures of hydrocarbons can also be independently or simultaneously introduced. The average intensity at the test sample is roughly 10 times the maximum intensity predicted on any optic in a commercial stepper. ${ }^{4}$ This allows limited scaling studies to investigate the dependence of damage rate on various adjustable quantities that could be used to accelerate the lifetime tests.

During an endurance exposure the ML mirror under test is placed at the focal spot of the beam and oriented to reflect the incident EUV radiation at 10 degrees. The reflectivity is monitored during exposures by a $\mathrm{Zr}$-coated silicon photodiode that can be rotated to measure the incident and reflected power. This in situ measurement, however, lacks the sensitivity to accurately measure the critical $1-2 \%$ changes in reflectivity at $13.5 \mathrm{~nm}$. Post-exposure reflectometry using a monochromator tuned to $13.5 \mathrm{~nm}$ must be performed to accurately detect these small changes. Although the $0.3 \%$ absolute uncertainty of the reflectometer at NIST is certainly sufficient for this task, the measurement beam is slightly larger than the exposure-spot dimensions. Hence, the samples are sent to the Lawrence Berkeley Labs reflectometer at the Advanced Light Source where reflectivity maps of the exposed spots can be made with 100- $\mu \mathrm{m}$ step sizes.

\subsection{Exposures of Ru-capped multilayers at the NIST synchrotron}

Figure 2 shows the reflectivity maps of several 10-hour exposures under varying conditions of ambient water vapor pressure and time-averaged EUV intensity. Both the high and low intensity exposures display a surprising trend in which the mirror degradation actually decreases with increasing partial pressures of water. This effect is most evident in the lower intensity exposures.

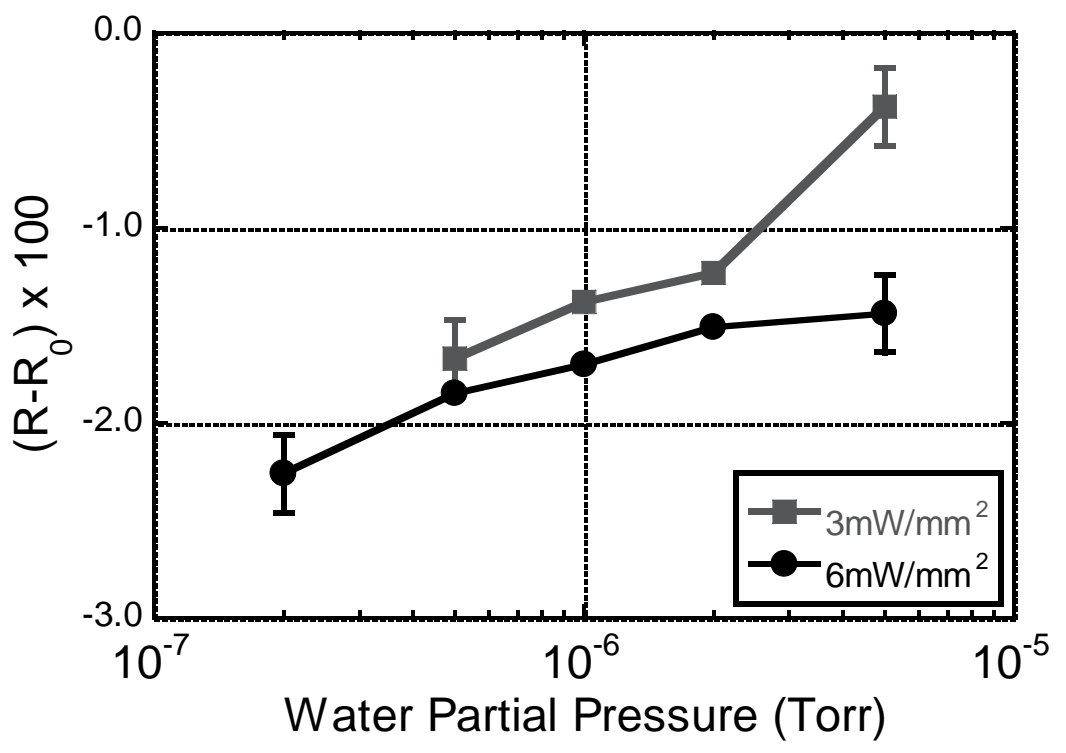

Figure 2. Change in reflectivity at the center of the exposure spots as a function of water partial pressure at two different average EUV intensities. Error bars represent combined uncertainty of absolute reflectivity measurements of exposed and unexposed $\left(\mathrm{R}_{0}\right)$ areas. 
One possible explanation of this unexpected result is that there is a mixture of water and hydrocarbon vapors in the exposure chamber. As demonstrated by Klebanoff, ${ }^{5}$ the damage caused by EUV $+\mathrm{H}_{2} \mathrm{O}$ can be significantly mitigated by admitting a mixture of ethanol and water at comparable partial pressures. It is thought that the $\mathrm{C}$ species produced by EUV cracking of the ethanol react with the potentially oxidizing $\mathrm{O}$ or $\mathrm{OH}$ radicals to form $\mathrm{CO}$ or $\mathrm{CO}_{2}$ which then leaves the surface. In this way the two potentially damaging reactions of carbon deposition and oxidation actually compete with each other producing much less damage to the mirror surface than either process alone.

Residual gas analysis (RGA) using a quadrupole mass spectrometer was performed to look for residual hydrocarbon contamination in our chamber. Figure 3 shows RGA spectra from the NIST test chamber with no admitted gasses (base pressure $8 \times 10^{-10}$ Torr) and with $5 \times 10^{-6}$ Torr of water. The base spectrum shows that the only significant peaks correspond to water $(\mathrm{m} / \mathrm{e}=18), \mathrm{CO}(\mathrm{m} / \mathrm{e}=28), \mathrm{CO}_{2}(\mathrm{~m} / \mathrm{e}=44)$ and $\mathrm{O}_{2}(\mathrm{~m} / \mathrm{e}=32)$. Additional scans out to $\mathrm{m} / \mathrm{e}=200$ show no peaks above the $10^{-12}$ Torr level for $\mathrm{m} / \mathrm{e}>44$. So the chamber itself is not a source of hydrocarbons under these conditions. The spectrum for admitted water is considerably more complicated, but the only peaks within a factor of 100 of the water peak are, once again, the $\mathrm{CO}, \mathrm{CO}_{2}$ and $\mathrm{O}_{2}$ peaks. More importantly, the characteristic signature of long-chain hydrocarbons (repeating clusters of peaks separated by $\mathrm{m} / \mathrm{e} \sim 14 \mathrm{CH}_{2}$ groups) is completely absent. So if the inverse dependence of mirror damage on water pressure is due to competition between oxidation and carbon deposition, the source of the $\mathrm{C}$ must be a simple, low mass C-compound. CO is the simplest such species and, from figure 3, is clearly present in our system. It could potentially adsorb onto the surface and react with surface $\mathrm{O}$ to form $\mathrm{CO}_{2}$, which is an extremely stable, essentially inert, molecule that would desorb without harming the surface.

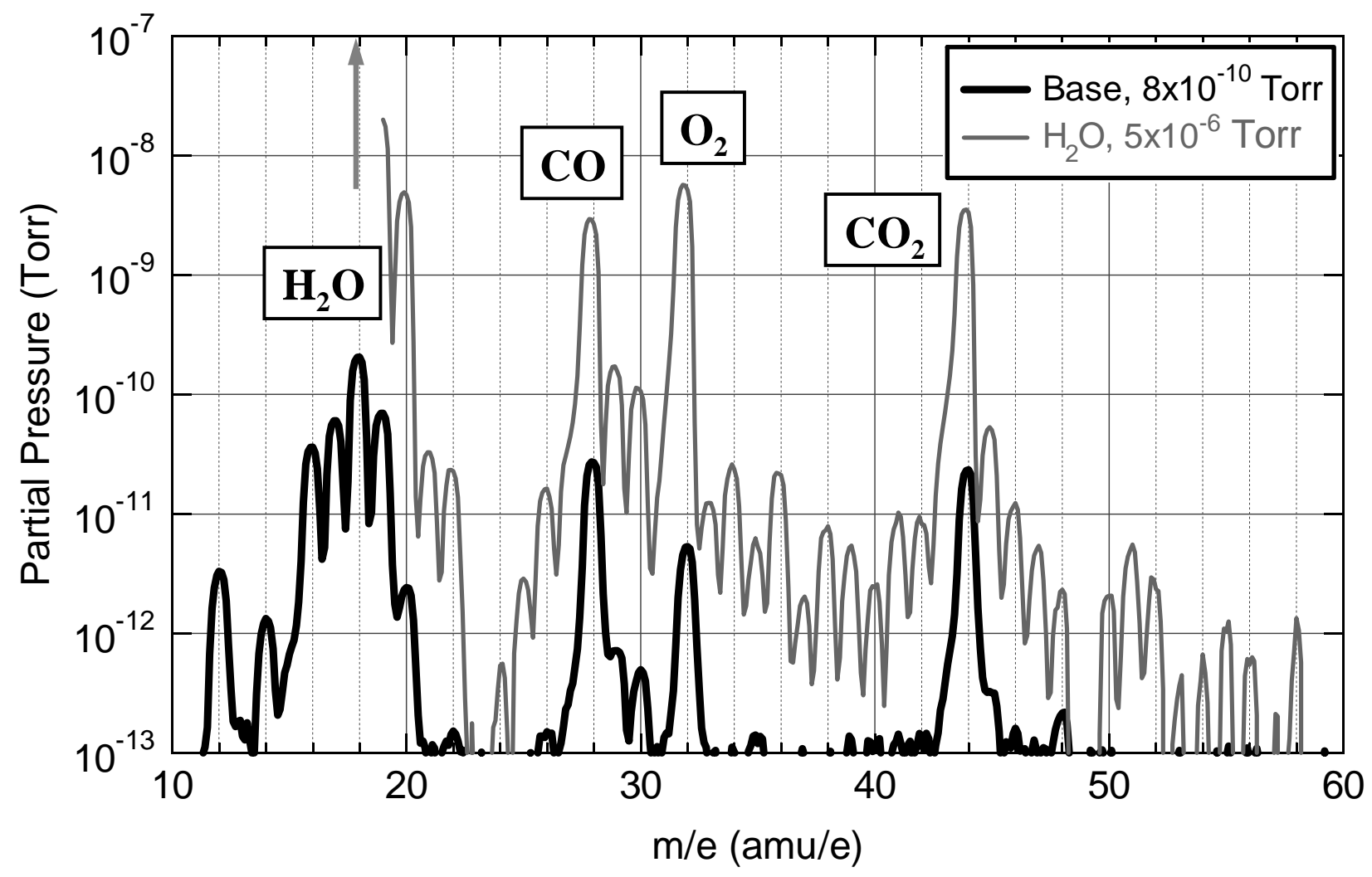

Figure 3. Residual gas spectrum of the base vacuum and of the chamber with $5 \times 10^{-6}$ Torr of admitted water vapor. Major peaks are labeled. The spectrum for the high partial pressure of water could not be recorded below $\mathrm{m} / \mathrm{e}=19$ due to saturation of the detector. Ancillary spectra verified that there were no significant peaks between $\mathrm{m} / \mathrm{e}=50$ and 200 for either case. 
While preparations were made to meet the hazardous-materials safety requirements of $\mathrm{CO}$, we investigated the sensitivity of the EUV $+\mathrm{H}_{2} \mathrm{O}$ damage rate to the partial pressure of admitted methanol, a more easily implemented Ccontaining species. (Tests with CO are currently underway.) Figure 4 shows the results of a series of exposures in water and methanol. Surprisingly the damage caused by 10-hr exposures in (0.5 and 1.0) $\times 10^{-6}$ Torr of water is completely mitigated by just $2 \times 10^{-8}$ Torr of methanol. While these results do not prove that CO would have the same effect, they do show that a few parts per thousand of some carbonaceous species can dramatically affect the EUV $+\mathrm{H}_{2} \mathrm{O}$ damage rate of ML mirrors. As discussed in the next section, this implies that measurement and control of the endurance testing vacuum environment is critically important.

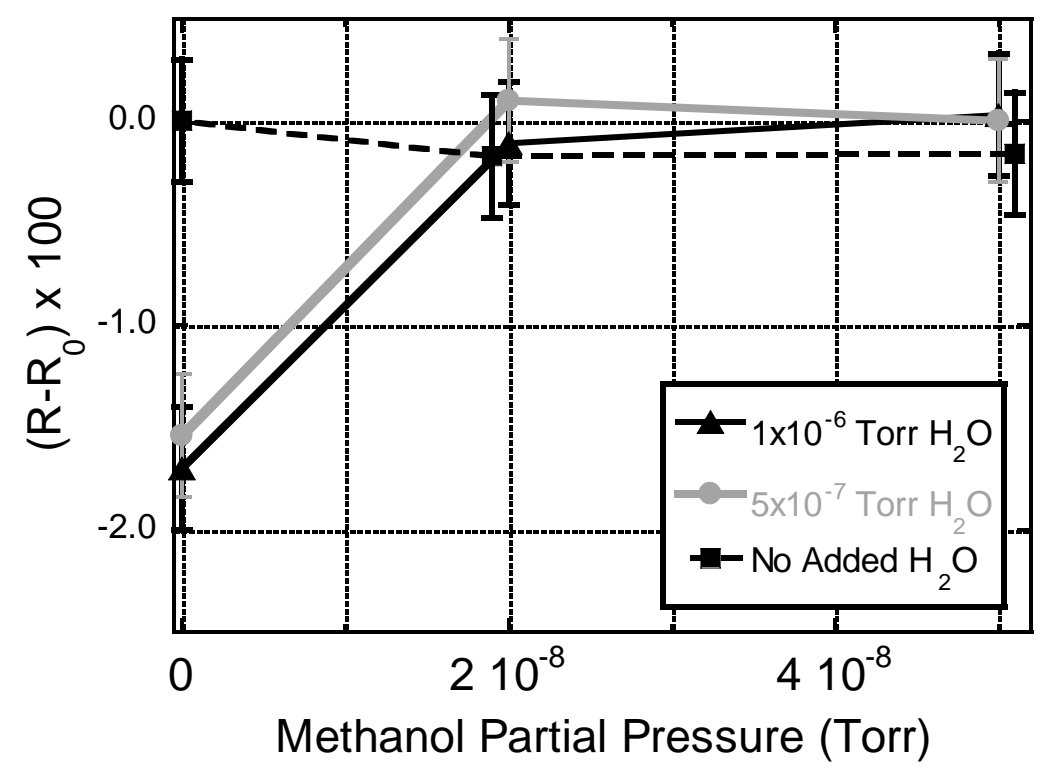

Figure 4. Peak reflectivity loss of $10 \mathrm{hr}$ exposures to $5 \mathrm{~mW} / \mathrm{mm}^{2}$ average EUV intensity with different levels of water and methanol. Error bars represent combined uncertainty of absolute reflectivity measurements of exposed and unexposed $\left(\mathrm{R}_{0}\right)$ areas.

\section{CRITICAL FACTORS FOR ENDURANCE TESTING}

\subsection{Vacuum environment}

The unexpected decrease in damage with increasing water pressure described above, led us to the possibility that the ML mirror damage rate can be significantly influenced by carbon-containing species present at partial pressures 100 to 1,000 times smaller than the water pressure. This implies that endurance or accelerated testing of ML mirrors could produce very misleading results unless the composition and relative constituent concentrations of the test chamber ambient vacuum are monitored and controlled to match the actual production environment on the parts-per-thousand level. Careful analysis of our own vacuum environment revealed that this is not simply a matter of achieving UHV conditions prior to admitting water vapor for endurance testing.

Since there is no evidence of long-chain hydrocarbon vapors in our chamber, we hypothesized that the increased levels of $\mathrm{CO}$ associated with admission of water (see RGA spectra in figure 3) might play a role in mitigating the damage caused by EUV $+\mathrm{H}_{2} \mathrm{O}$. There are two potential sources for the CO: 1) CO dissolved in the water supply; or 2) CO released from interactions between the admitted water and the vacuum chamber walls and/or filaments. The first source seemed the most likely, but relatively simple tests revealed that the ratio of the water and CO RGA peaks depends linearly on the pumping speed of the chamber as both pumping speed and the water leak rate are varied. This ratio would have been constant if the CO were dissolved in the water. Instead it was proportional to pumping speed, indicating that the $\mathrm{CO}$ is the result of water interacting with some carbonaceous species in the chamber itself. Such 
interactions are a common phenomenon that are well known and carefully avoided in surface physics. ${ }^{6}$ The most likely reactions affecting our vacuum environment are 1 ) the displacement/desorption of otherwise non-volatile carbonaceous species by the dissociative adsorption of $\mathrm{H}_{2} \mathrm{O}$ onto the stainless steel walls of the vacuum chamber; and 2) the direct formation of $\mathrm{CO}$ when water reacts with the ubiquitous carbon contamination in the hot filaments of ion gauges and quadrupole mass spectrometers. To avoid these and other effects when a surface must be exposed to a large dose of a pure gas (e.g., water vapor), surface scientists routinely use a "doser" to roughly collimate a beam of gas through a capillary array capable of delivering a high flux onto the surface while minimizing the increase in the ambient partial pressure. $^{7}$

Clearly, the planning and execution of accelerated testing under aggressive conditions are greatly complicated by the extreme sensitivity of the mirror-damage rate to trace levels of residual C-containing gasses, and the unwanted production of these gasses at elevated pressures of water. NIST plans to address these issues by using a doser to reach high effective pressures at the sample but not at the chamber walls. Also, since water and most other gasses do not readily adsorb onto gold at room temperature, we have gold plated as many of the vacuum surfaces as possible to reduce wall interactions.

\subsection{Wavelength considerations}

The EUV-induced oxidation and carbonization of the optics can arise from several processes. ${ }^{8}$ While EUV photodissociation is possible, collisional dissociation of adsorbed water vapor and carbonaceous species by secondary electrons is thought to be the more likely reaction. In the absence of resonances, one would not expect either of these processes to have a strong dependence on EUV wavelength; however, the constructive interference responsible for producing the high reflectivity of a ML mirror does introduce marked wavelength effects for small deviations from the design wavelength.

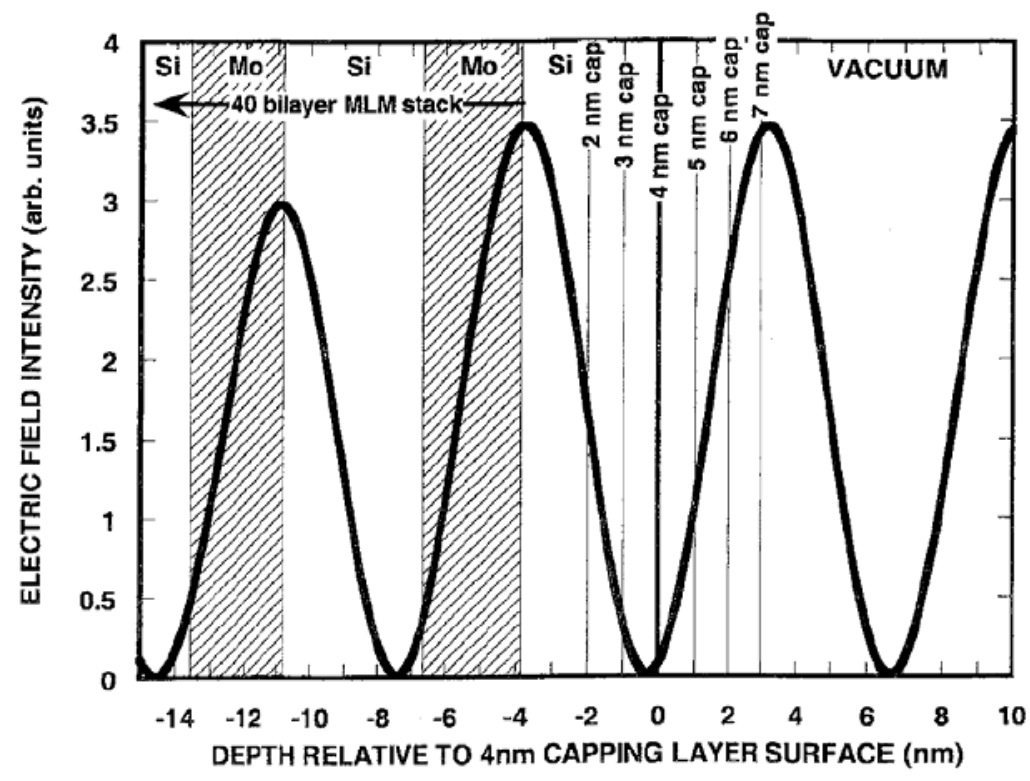

Figure 5. Calculated results for electric field intensity near the surface of a MoSi multilayer mirror. Normally the cap layer thickness (in this case 4-nm $\mathrm{Si}$ ) is chosen so that the intensity is minimum at the surface. (Figure reprinted with permission from Reference 9.) 
Figure 5 shows the calculated electric field intensity near the surface of a 40-period MoSi ML irradiated with resonant 13.5-nm radiation. (Figure reprinted with permission from reference 9.) For the resonant wavelength the intensity is a standing wave with a 7-nm period. The calculation was done for a Si cap layer, but the results for Ru or any other material would not be qualitatively different. Normally, the cap layer thickness is chosen so that the standing wave has a minimum at the surface of the layer, in this case $\sim 4 \mathrm{~nm}$.

Figure 6, also taken from the same work, shows the measured photoemission from ML mirrors with different thicknesses of a Si capping layers plotted against the electric field intensity taken from figure 5. These results clearly demonstrate that the photoemission intensity strongly depends on the electric field intensity at the surface and thus on the position of the surface plane relative to the standing wave. Since the oxidation and carbonization processes are induced by either the secondary electrons or the primary photons, the rate of reflectivity loss should likewise vary with the intensity at the surface. Experiments performed by the authors of reference 9 show strong correlation between secondary photoemission rate and the rate of reflectivity loss due to carbon build up as the carbon layer increased in thickness for the first few nanometers. These results corroborate the connection between field intensity at the surface and the rate of photo-induced surface reactions.

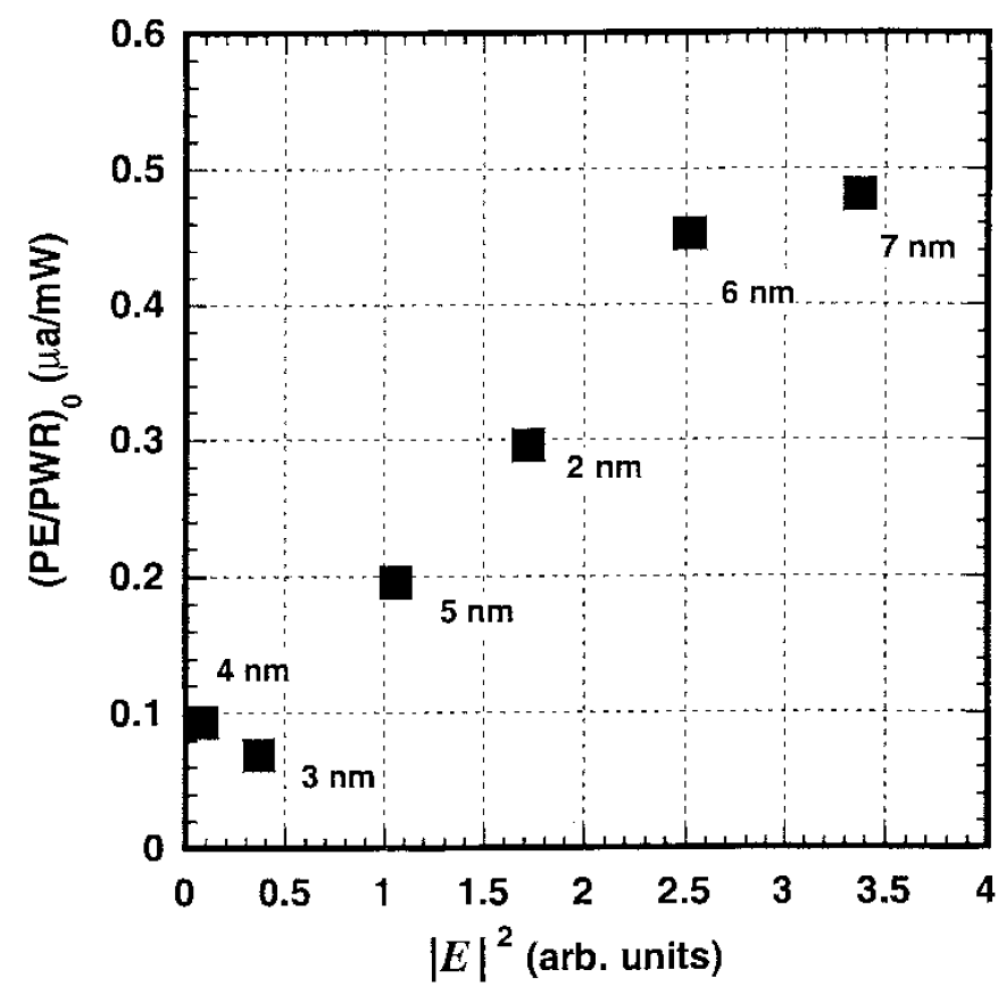

Figure 6. Correlation between measured photoemission and calculated electric field intensity for different thicknesses of Si capping layers. (Figure reprinted with permission from Reference 9.)

This resonant standing wave behavior of the EUV mirrors means that endurance tests must either be done at the design wavelength or that a model must be invoked to account for the difference in field intensity at the ML surface connected with the non resonant irradiation. For example, exposure tests performed using grazing incidence mirrors to collect a spectral band limited by a Be or Zr filter might actually be subjecting the sample surface to higher field intensities than exposure tests performed with resonant 13.5-nm radiation with the same total intensity. 


\subsection{Intensity profile spatial effects}

Figure 7 shows an example of an effect that contradicts the notion that areas illuminated at higher intensity and therefore accumulating a greater dose would suffer greater loss of reflectivity. In the figure we see that the reflectivity profile of a spot exposed by a 650x650- $\mu \mathrm{m}$ Gaussian EUV beam actually has less reflectivity loss at the center where the beam is most intense and the dose is highest. This anomalous effect has been seen to varying degrees in all exposures thus far lasting 40 hours or more.

One possible explanation is connected with the surface diffusion of weakly bound molecules into the small region of the exposed spot. In a model that could explain the effect, the EUV depletes the molecules in the illuminated spot by either photon-stimulated desorption or photon-stimulated dissociation into a reactive species. This depletion results in a surface-coverage gradient that causes net surface diffusion of molecules into the depleted area. The fact that less damage occurs at the center indicates that this area is not replenished by the surface diffusion as effectively as the surrounding area because a major fraction of the diffusing molecules are either desorbed or dissociated at the intermediate intensities surrounding the central spot. It is also possible that the highly non-uniform damage distribution is produced by the interaction of the competing carbonization and oxidizing reaction kinetics in the presence of a strong intensity gradient.

As with precautions one must take to ensure good control of trace background contamination and wavelength, we believe that one must also take into account that results from tests using spot illumination may not be truly representative of what can be expected of mirror behavior in the tool environment where the entire mirror surface is uniformly illuminated.

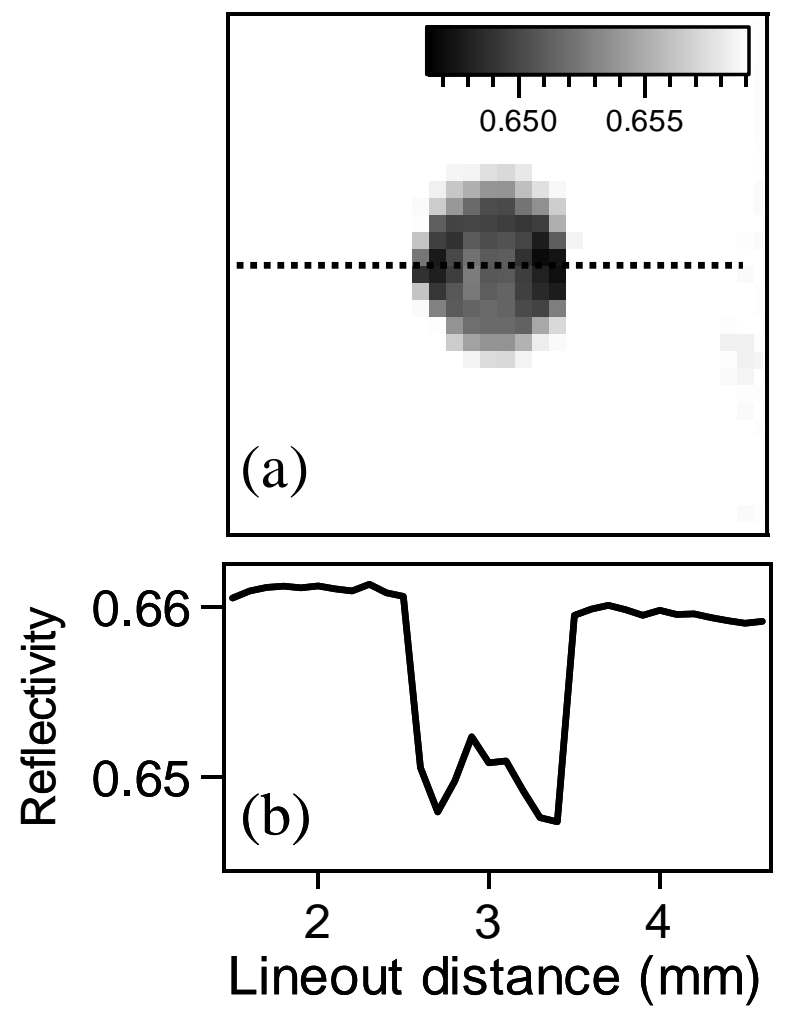

Figure 7. (a) Reflecivity map showing non-Gaussian damage profile typical of exposures lasting longer than 40hrs at $\sim 5 \mathrm{~mW} / \mathrm{mm}^{2}$. (b) Lineout of reflectivity data along path indicated by dotted line in (a). 


\section{SUMMARY}

Advancing EUV metrology in support of the commercialization of EUVL has been a NIST high priority since the early 1990s. The NIST capabilities include radiometric calibrations of detectors, filter-detector packages and spectrometers; accurate reflectometry; and long-term EUV mirror endurance testing. Recent endurance testing results imply that the damage to Ru-capped multilayer mirrors exposed to EUV in the presence of elevated partial pressures of water can be significantly affected by a few parts-per-thousand of carbon-containing background gasses. High-spatial-resolution reflectometry of large-dose exposures ( $>40 \mathrm{hrs}$ at $\sim 5 \mathrm{~mW} / \mathrm{mm}^{2}$ ) also revealed a highly non-linear correlation between the spatial distribution of mirror damage and the Gaussian EUV intensity profile of the exposure beam. This suggests that surface diffusion or other competing reaction kinetics may significantly influence the results of tests performed with a tightly focused EUV beam. Additional experiments are currently underway to further verify these effects. Such factors must be well understood and accounted for before results from endurance or accelerated testing can be used to project the lifetime of optics in the production environment.

\section{REFERENCES}

${ }^{1}$ L. R. Canfield and N. Swanson, “Far ultraviolet detector standards”, J. Res. NBS 92, 97-112 (1987).

${ }^{2}$ C. Tarrio, R. N. Watts, T. B. Lucatorto, M. Haass, T. A. Calcott, and J. Jia, "New NIST/DARPA National Soft X-ray Reflectometry Facility", J. X-ray Sci. Tech. 4, 96 (1994).

${ }^{3} \mathrm{C}$. Tarrio and S. Grantham, "A synchrotron beamline for extreme-ultraviolet multilayer mirror endurance testing”, submitted to Rev. Sci. Instrum.

${ }^{4}$ S. Bajt, H.N. Chapman, N. Nguyen, J. Alameda, J.C. Robinson, M. Malinowski, E. Gullikson, A. Aquila, C. Tarrio and S. Grantham, "Design and performance of capping layers for extreme-ultraviolet multilayer mirrors", Applied Optics 42, 5750 (2003).

${ }^{5}$ L.E. Klebanoff, M.A. Malinowski, W.M. Clift, C. Steinhaus, and P. Grunow, "Use of gas-phase ethanol to mitigate extreme UV/water oxidation of extreme UV optics”, J. Vac. Sci. Technol. A22(2), 425 (2004).

${ }^{6}$ T. E. Madey, "Summary Abstract: Surface phenomena and their influence on ultrahigh vacuum gauges," J. Vac. Sci. Technology A5, 3249 (1987)

7 J. T. Yates, Jr., "Experimental innovations in surface science", AIP Press, Springer, New York, 1998.

${ }^{8}$ T. E. Madey, N. S. Faradzhev, B. V. Yakshinskiy and N. V. Edwards "Surface phenomena related to mirror degradation in extreme ultraviolet (EUV) lithography" Appl. Surf. Sci. (2006) in press

${ }^{9}$ M. Malinowski, C. Steinhaus, M. Clift, L.E. Klebanoff, S. Mrowka, and R. Soufli, "Controlling Contamination in Mo/Si Multilayer Mirror by Si Surface-capping Modifications”, Proceeding of SPIE, 4688, 442 (2002). 